\title{
Situación de la gestión curricular en programas de enfermería en tiempos de pandemia por Covid-19
}

\author{
Situation of curricular management in nursing programs in times of the Covid-19 pandemic
}

Situação da gestão curricular nos cursos de enfermagem em tempos de pandemia de Covid-19

\section{ARTÍCULO GENERAL}

Mg. Mistral Ena Carhuapoma Acosta

https://orcid.org/0000-0001-9363-3509

mcarhuapomaa@unmsm.edu.pe

Universidad Nacional Mayor de San Marcos

\author{
Dra. Angela Rocio Cornejo Valdivia \\ https://orcid.org/0000-0003-2658-7033 \\ acornejov@unmsm.edu.pe
}

Universidad Nacional Mayor de San Marcos

Recibido 01 de Noviembre 2021 | Arbitrado y aceptado 01 de Noviembre 2021 | Publicado en 24 Diciembre 2021

\section{RESUMEN}

El presente artículo se propuso como objetivo reflexionar sobre la situación panorámica de la gestión curricular en programas de enfermería en el contexto de la pandemia por Covid-19, ello a través de la revisión de la literatura de los últimos años y el respectivo análisis de los artículos identificados. Para ello, como se menciona, se hizo uso de una metodología basada en la revisión bibliográfica consultando bases de datos como Proquest, Scopus y Google Scholar para encontrar revistas del más alto nivel. Asimismo, se emplearon palabras clave como Gestión curricular, enfermería, pandemia, programas de enfermería. Algunos de los criterios o filtros que se utilizaron fueron: que sean artículos de revistas, y la antigüedad de la publicación que no sea mayor a 5 años y de los últimos dos en casos de Covid19. Con todo ello, se pudo presentar los resultados por categorías temáticas de acuerdo con la información recabada de los documentos. Así, se tiene un recorrido por problemáticas relevantes y vigentes como el panorama de la educación en tiempos de pandemia por Covid-19, y especificando en la disciplina de la enfermería, las nociones básicas de la gestión y la gestión curricular y el rol de esta en los programas de enfermería así como los cambios que se han venido dando y los que pueden darse en la currícula de esta carrera. Se mencionan entre las incorporaciones más relevantes el uso combinado de modelos sincrónicos y asincrónicos, las tecnologías de información y comunicación y la revalorización de la simulación clínica.

Palabras clave: Gestión curricular, enfermería, pandemia, currículo programas de enfermería.

\section{ABSTRACT}

The objective of this article was to reflect on the panoramic situation of curricular management in nursing programs in the context of the Covid-19 pandemic, through a review of the literature of recent years and the respective analysis of the identified items. For this, as mentioned, a methodology based on bibliographic review was used, consulting databases such as Proquest, Scopus and Google Scholar to find journals of the highest level. Likewise, keywords such as curricular management, nursing, pandemic, nursing programs were used. Some of the criteria or filters that were used were: that they be journal articles, and the age of the publication that is not more than 5 years and the last two in cases of Covid-19. With all this, it was possible to present the results by thematic categories according to the information collected from the documents. Thus, there is a tour of relevant and current problems such as the panorama of education in times of the Covid-19 pandemic, and specifying in the discipline of nursing, the basic notions of management and curricular management and the role of this in nursing programs as well as the changes that have been taking place and those that may occur in the curriculum of this career. Among the most relevant additions, the combined use of synchronous and asynchronous models, information and communication technologies and the revaluation of clinical simulation are mentioned.

Keywords: Curriculum management, nursing, pandemic, nursing programs curriculum.

\section{RESUMO}

O objetivo deste artigo foi refletir sobre a situação panorâmica da gestão curricular nos cursos de enfermagem no contexto da pandemia de Covid-19, através de uma revisão da literatura dos últimos anos e da respetiva análise dos itens identificados. Para isso, conforme mencionado, foi utilizada uma metodologia baseada em revisão bibliográfica, consultando bases de dados como Proquest, Scopus e Google Acadêmico para encontrar periódicos do mais alto nível. Da mesma forma, foram utilizadas palavras-chave como gestão curricular, enfermagem, pandemia, programas de enfermagem. Alguns dos critérios ou filtros utilizados foram: que sejam artigos de periódicos, e a idade da publicação que não seja superior a 5 anos e os dois últimos em casos de Covid-19. Com tudo isso, foi possível apresentar os resultados por categorias temáticas de acordo com as informações coletadas nos documentos. Assim, faz-se um passeio por problemas relevantes e atuais como o panorama da educação em tempos de pandemia da Covid-19, e especificando na disciplina de enfermagem, as noções básicas de gestão e gestão curricular e o papel desta nos cursos de enfermagem bem como as mudanças que vêm ocorrendo e as que podem ocorrer no currículo desta carreira. Entre os acréscimos mais relevantes, são mencionados o uso combinado de modelos síncronos e assíncronos, tecnologias de informação e comunicação e a reavaliação da simulação clínica.

Palavras-chave: Gestão curricular, enfermagem, pandemia, currículo dos programas de enfermagem. 


\section{Introducción}

Mg. Mistral Ena Carhuapoma Acosta, Dra. Angela Rocio Cornejo Valdivia

En esta denominada "Pandemia de la globalización", el sector salud ha sido la institución que más se ha visto rebasada, en cuanto a los recursos humanos, insumos, investigación e infraestructura hospitalaria. Debido a la naturaleza de la práctica del profesional de salud, el personal de enfermería específicamente se ha visto disminuido por enfermedades previas, nuevos contagios y desafortunados decesos, además de que los insumos resultaron insuficientes en diversas etapas de la contingencia. En este marco de emergencia sanitaria e institucional, el sector educativo también se ha visto en la necesidad de realizar cambios radicales. En esta misma línea de ideas, los estudiantes del sistema de educación en enfermería se han visto en la necesidad de adaptarse a esta ola de cambios vertiginosos, en primer lugar en las instituciones donde pasan la mayor parte de su tiempo, en segundo lugar en la familia y en tercer lugar y no menos importante en su profesionalización (Zárate et al., 2020).

La formación de profesionales en enfermería ha constituido una dimensión clave en torno a la calidad del proceso enseñanza-aprendizaje, que en la actualidad se agudiza debido a los requerimientos surgidos por la globalización y la sociedad del conocimiento (J. Sánchez et al., 2019) pero, sobre todo, a causa del momento que atraviesa la sociedad hoy, indicado por los epidemiólogos como uno de los mayores desafíos sanitarios a escala mundial de este siglo: la pandemia de Covid-19 (Costa et al., 2020); así, la sociedad requiere profesionales acordes con las nuevas tendencias y competentes para transformar la realidad para el beneficio común (Ayala \& Espinoza, 2020).

Al respecto, la Escuela de Enfermería es una organización compleja que gestiona la enseñanza y el aprendizaje de la disciplina de la Enfermería, fenómenos dinámicos que no pueden verse separados de otros aspectos que están involucrados en la administración de recursos como son la planta física, el acceso a las tecnologías informáticas, la cantidad y calidad del personal docente y administrativo, los recursos financieros, entre otros (Guzman, 2013).

Pero con el contexto actual, cabe preguntarse ¿Cómo está afectando la pandemia a la gestión curricular en la disciplina de la enfermería? ¿De qué maneras la Enfermería puede/necesita reinventarse? Y, ¿Qué es lo fundamental que debe preservarse con respecto a la currícula en el proceso de formación de los enfermeros? (Costa et al., 2020). 
El proceso de gestión curricular, entendido como la acción colectiva que involucra a todos los actores que intervienen en el proceso de formación, tiene como propósito articular estos elementos para lograr el mejor resultado por lo que es preciso indagar y dar seguimiento a las necesidades e inquietudes de los actores así como a la evolución de los asuntos organizativos (Guzman, 2013). Ello se vincula, además, a la problemática de los espacios educativos convertidos en un frío escenario, donde se requiere conseguir la interacción docente-alumno para proyectar la verdadera esencia del saber (Speranza et al., 2020).

Guzman, 2013 afirma que el currículo es una acción deliberativa entre los participantes, (docentes, estudiantes, administrativos) que mediante consenso y argumentos sólidos logran ir construyendo y reconstruyendo la propuesta, de manera que responda en buena medida a los problemas que viven los y las profesionales en Enfermería.

Así, Zárate et al., 2020 identifica y prevé la necesidad de establecer algunos cambios en esta currícula que permitan que la formación de especialización en los diversos campos de conocimiento vayan acorde con las circunstancias actuales, a la vez que prevea los diversos escenarios que irán surgiendo en el transcurso.

La fuente previa indica que algunos de los cambios que se visualizan como indispensables pueden ser la reorientación del tipo e instrumentación de contenidos curriculares que redireccionen el ejercicio profesional considerando las circunstancias actuales. Así también, el quehacer de la enseñanza-aprendizaje en los tiempos de pandemia ha implicado la creación, como en otras disciplinas, de un aula virtual introduciendo, incluso, una tendencia educativa llamada aprendizaje ubicuo, una tecnología que potencializa el aprendizaje en todo lugar y en cualquier momento, todo esto utilizado a favor del aprendizaje, trabajo colaborativo, intercreatividad y la sabiduría de las multitudes.

Este proceso, no obstante, es largo y requiere de un consejo en la carrera de enfermería que coteje coherencia y consistencia de los cambios en la malla curricular y la gestión de la formación de los alumnos a través del diseño de una metodología y su posterior aplicación para evaluar si responde al perfil de egreso establecido, asegurando la calidad del proceso de aprendizaje institucional. 
Por todo ello, la presente se plantea cuestionamientos, por ejemplo, ¿Cómo se ha afectado la formación de enfermería?; ¿Que caracterizaba a los modelos de gestión curricular ante de la pandemia?; ¿Cómo debería cambiar o replantearse la gestión en los tiempos actuales? En ese sentido, se propone como objetivo reflexionar sobre la situación panorámica de la gestión curricular en programas de enfermería en el contexto de la pandemia por Covid-19 en la medida que se pueda contribuir con información que apoye a una formación en enfermería acorde a las necesidades de la sociedad actual; todo ello a través de la revisión de la literatura de los últimos años y el respectivo análisis de los artículos identificados.

\section{Metodología}

La búsqueda de la información para la revisión de literatura se hizo consultando las siguientes bases de datos como son Proquest, Scopus y Google Scholar para encontrar revistas del más alto nivel científico. Asimismo, cabe mencionar que para la realización de lo mencionado se emplearon también palabras clave como como Gestión curricular, enfermería, pandemia, programas de enfermería.

Cada uno de estos descriptores se combinaron entre sí durante la búsqueda utilizando los operadores boléanos "and" y "or". Además, algunos de los criterios o filtros que se utilizaron para hacer muchos más específica la búsqueda y ser más precisos con los documentos encontrados en las diferentes bases de datos consultadas fueron: que sean artículos de revistas, y la antigüedad de la publicación que no sea mayor a 5 años y del último año en casos de Covid-19.

Toda la información previa relacionada a los filtros aplicados y las especificaciones sirvieron como criterios de inclusión, es decir que sean artículos publicados en revistas científicas, que no excedan los cinco años de antigüedad, que la temática guarde relación con las variables de gestión curricular de enfermería y tiempos de pandemia por Covid19. Se excluyeron, en tanto, todos los documentos que no respetaran lo postulado así como aquellos que estaban incompletos o que tenían enlaces averiados.

\section{Resultados y discusión}

\section{Educación en tiempos de pandemia}

En tiempos de pandemia, de temer que la educación y la cultura sean víctimas de la austeridad de muchos gobiernos, quienes privilegiarán la reactivación de los sectores 
económicos (Feyen, 2020), y si a ello se le suman las diversas problemáticas ya existentes, deja un panorama un tanto desolador.

Velazque et al., 2020 señaló que las universidades en el Perú (así como en diversas partes del mundo) se vieron en la obligación de cerrar sus puertas, interrumpir las clases e incluso los estudiantes y docentes que cursaban pasantías e intercambios a nivel nacional e internacional fueron cancelados al no poder ser evaluados, y representó gran dificultad para retornar a sus lugares de origen.

Además, aspectos como la suspensión de las actividades docentes presenciales para dar paso a la virtualización supuso que el profesorado y el alumnado tengan una elevada presión, tanto en su trabajo como en la adaptación al nuevo escenario (Tejedor et al., 2020). Igualmente, la transición a esta docencia online ha sido abrupta, desconociendo los largos procesos de formación, de rediseño de asignaturas y de infraestructuras que soporten la demanda de la tele-enseñanza (González et al., 2020).

De hecho, en varios países latinoamericanos, la Covid-19 influyó negativamente en el proceso de enseñanza-aprendizaje, debido a que no se invirtió durante muchos años en la adecuación de los campus virtuales, los sitios web institucionales, las revistas científicas digitales y en la capacitación de los docentes y alumnos en el manejo de las TICs, teniendo que improvisar soluciones tecnológicas (Ríos, 2020).

En el Perú, específicamente, (Velazque et al., 2020) precisa que hay severas limitaciones, por lo complicado que resulta para muchos docentes realizar cursos a distancia y de manera fluida, efectiva e interactiva, especialmente, como también señala (Ríos, 2020), esto es más visible en las universidades públicas del país.

Por otra parte, se considera también las limitaciones tecnológicas que pueda presentar el estudiante, considerando que muchas universidades públicas albergan a estudiantes de provincias y de nivel socioeconómico bajo y medio, teniendo como mínimo sólo los servicios básicos, no contando todos ellos por ejemplo con una computadora o servicio de internet eficiente y continuo o tal vez de espacios físicos disponibles.

Ello se relaciona a lo investigado por (Huanca et al., 2020) quienes demostraron que de una participación de estudiantes de universidades de gestión pública y privada, el $55 \%$ de los estudiantes afirmó contar con acceso a internet mientras que el $45 \%$ no cuenta con ello. La situación se agrava al considerar únicamente la participación de estudiantes 
de universidades de gestión pública (estatales) ubicadas en la ciudad de Lima y provincias. Según los resultados, el $68 \%$ refirió no tener acceso a internet y solo un $32 \%$ afirma que sí lo tiene.

De esta forma, (Tejedor et al., 2020) coinciden en que la Covid-19 ha evidenciado la urgente transformación que demandan los sistemas educativos tradicionales y la importancia de poseer una estrategia educativa virtual, así como un alumnado y un profesorado con habilidades y competencias para la enseñanza y el aprendizaje en el ciberespacio. La irrupción de este virus planetario ha dejado constancia de las exigencias de una pandemia a las instituciones educativa: flexibilidad, plataformas, metodologías y contenidos adaptados a unos intercambios formativos mediados por las pantallas.

Si bien son tiempos complejos, son estas circunstancias las que ofrecen un espacio de apertura al cambio, a la innovación, a la creatividad y a mirar este proceso con la perspectiva más humana posible (Jaque, 2020).

\section{Educación de enfermería en tiempos de pandemia}

Teniendo como base el contexto descrito previamente, y más aún debido a que la profesión de enfermería ha tomado un rol preponderante en el equipo de salud que lidia con dicha problemática de nivel pandémico, se ha visto profundamente afectada por esta crisis y se ha puesto en evidencia inequívoca la falta de inversión para fortalecer su quehacer profesional y especializado (Jaque, 2020); (Cassiani et al., 2020).

Ya que el aislamiento social es necesario y la misión actual es la protección a la vida, cabe cuestionarse ¿cómo va a ser la formación de los estudiantes de Enfermería en este contexto? Las escuelas de enfermería de todo el mundo han reemplazado la enseñanza teórica y práctica presencial con alternativas en línea. Sin embargo, muchos educadores, familiarizados con el modelo clásico de capacitación cara a cara, tenían dudas sobre la efectividad de la tecnología en la enseñanza y el aprendizaje en materias complejas de enfermería y atención médica. Además, el problema previamente mencionado respecto a la disponibilidad actual de recursos y herramientas que permitan una metodología de vanguardia. Otro problema es la no existencia de una vasta experiencia para garantizar el éxito, ni tampoco garantizar el acceso generalizado de los estudiantes a un computador e Internet (Marques, 2020).

Es en la adversidad donde nace la fuerza, por lo que, tal y como decía (Jaque, 2020) u de acuerdo también con (Costa et al., 2020) el revés de la pandemia consiste 
precisamente en movilizar a las personas, a las instituciones y al poder público para repensar los caminos que se han tomado en el campo de la educación superior, la ciencia y la tecnología, así como la propia función social e intersectorial de la Enfermería.

De acuerdo con Carmen Morales, directora del Programa de Neurociencias, Sistema de Salud Menonita, en el reporte de (Elseiver \& R\&A Smarth Health, 2020) el coronavirus ha llegó para quedarse, y "es algo que tenemos que asumir y con lo que vamos a tener que convivir” (p. 25). Además, y coincidiendo con (Tejedor et al., 2020) señaló que es más que probable que el ser humano tenga que hacer frente a nuevas pandemias, por lo que es muy importante dotar a los profesionales de la enfermería de las herramientas necesarias para hacer frente a las exigencias de este nuevo escenario.

\section{Gestión Curricular}

Se entiende por gestión el conjunto de actividades que se llevan a cabo de forma coordinada para alcanzar lo establecido dentro de un proceso de planificación. Incluye aquellas actividades que contribuyen al logro de objetivos y metas e implica un mejor uso de los recursos. Además, busca transformar una situación en otra. De ahí la importancia de orientar los esfuerzos para lograr lo propuesto, tomar decisiones dentro del proceso, medir lo realizado y compararlo con lo esperado (Ángel \& Lopera, 2013).

En enfermería, según la fuente previa, la gestión se identifica como un proceso humano y social encaminado a movilizar a las personas y los recursos del entorno para mantener y favorecer el cuidado. También se concibe como la aplicación del juicio profesional en la planificación, organización, motivación y control del cuidado, de manera oportuna, segura e integral, que aseguren la continuidad de la atención y se apoyen en las políticas y lineamientos estratégicos de la institución

Asimismo, se define la gestión curricular como un proceso participativo que involucra a un colectivo de docentes en una institución educacional, con el fin de propiciar reformas sistemáticas del plan de estudio, dirigido a garantizar su adecuación al entorno de la entidad y sustentado en una actitud pertinente del colectivo ante el cambio (V. Sánchez et al., 2016). Es, además, entendido como un proceso académico que representa una herramienta de toma de decisiones curriculares relevante en los programas, ello incluye acorde al contexto que se tiene en la actualidad, los programas de modalidad a distancia (Benavidez \& Vásquez, 2019). 
Para López, 1997 citado en Benavidez \& Vásquez, 2019 se reconoce la gestión curricular, como la construcción de espacios de libertad conceptual y teórica, pero con autonomía institucional, la cual determina el derrotero desde el modelo pedagógico, pretende dar respuesta a preguntas como:

- ¿qué se debe enseñar?

- ¿cómo se lograría el aprendizaje?

Ello hace posible la fusión de modelos cognitivos, que identifiquen el objeto de saber, presentando una percepción de la realidad del programa a diseñar, permitiendo la trascendencia cultural y la prevalencia de su contexto desde el área de conocimiento aplicado.

La gestión curricular es un constructo multidimensional, determinado por un gran número de variables (inteligencia, motivación, personalidad, etc.) y en el que influyen numerosos factores personales, familiares o escolares, entre otros (Benavidez \& Vásquez, 2019).

\section{Principios de la gestión curricular}

De acuerdo con (Álvarez \& Díaz, 2019) los principios orientadores de la gestión curricular, se puede decir que ella se centra en cuatro aspectos fundamentales que son:

a. El diseño curricular. Entendido como la capacidad de los docentes, directores, padres de familia, estudiantes, organismos sociales y organismos políticos nacionales e internacionales, por hacer propuestas, planes curriculares, y programas de formación, con fines totalmente articulados, respetuosos, estudiados crítica y objetivamente, para ser puestos en marcha con la convicción de ser soluciones a las problemáticas sociales que allí se suscitan. Por el compromiso social que tiene como objetivo la educación con estas realidades humanas, es que se deben planear y desarrollar propuestas curriculares que cumplan satisfactoriamente con las necesidades de la población y de los alumnos que han sido inscritos en ellas.

b. La gestión del currículo: entendiéndola como la capacidad de todos los agentes involucrados en el proyecto educativo institucional, el currículo y el programa educativo, de cumplir con todos los requerimientos presupuestales, técnicos, administrativos, organizativos y los demás necesarios para cumplir con el cometido propuesto en el plan y con el programa educativo. 
c. La evaluación permanente del currículo: a través de evaluaciones de resultado obtenidos por la institución educativa; en el que se deben observar de manera objetiva el impacto que ha propiciado el currículo al interior de la misma, así, como también y de manera más importante; si el currículo ha favorecido a quien ha permitido ser parte de su formación o de quien hace parte de su creación.

d. El rediseño curricular: el cual también exige un análisis permanente de las fortalezas y debilidades educativas, de las mejores prácticas que orienten el proceso de enseñanza aprendizaje, de las características del entorno, de las necesidades sociales, del impacto laboral, de la oferta y demanda de perfiles ocupacionales y de las circunstancias políticas y económicas por las que atraviese el país en el momento.

\section{Gestión curricular de enfermería en tiempos de pandemia}

Es preciso indicar que la formación curricular de la enfermera durante la crisis de la pandemia por Covid-19 demanda fortalecerle en aspectos relacionados con la autonomía, toma de decisiones, ejercicio ético e innovación de su práctica profesional ante nuevos escenarios, a fin de impulsar la transformación de su rol y reconocimiento social, por el desempeño ejercido en eventos emergentes pasados y el actual (Zárate et al., 2020).

Asimismo, apuntar hacia una educación de calidad, más necesaria que nunca, para la promoción de una nueva generación de graduados que haga frente a los múltiples desafíos del siglo XXI, jóvenes que trabajen hacia una distribución más equitativa del bienestar y conviertan a la sociedad en un lugar más habitable y pacífico, no solo para unos pocos, sino para todos (Feyen, 2020); (Tejedor et al., 2020).

\section{Modelos y etapas en la gestión curricular}

Benavidez \& Vásquez, 2019 precisa que se pueden identificar en la literatura la existencia de diversos modelos de gestión que presentan diferentes estructuras y etapas, así también generan otros procesos que permiten a la entidad profundizar en la gestión del currículo en las instituciones de educación superior. 
Mg. Mistral Ena Carhuapoma Acosta, Dra. Angela Rocio Cornejo Valdivia

Por ejemplo, la fuente previa citando a Tobón, 2008 presentan el siguiente modelo con la estructura que se ve en la Figura 1.

Figura 1. Estructura de la gestión curricular según Tobón

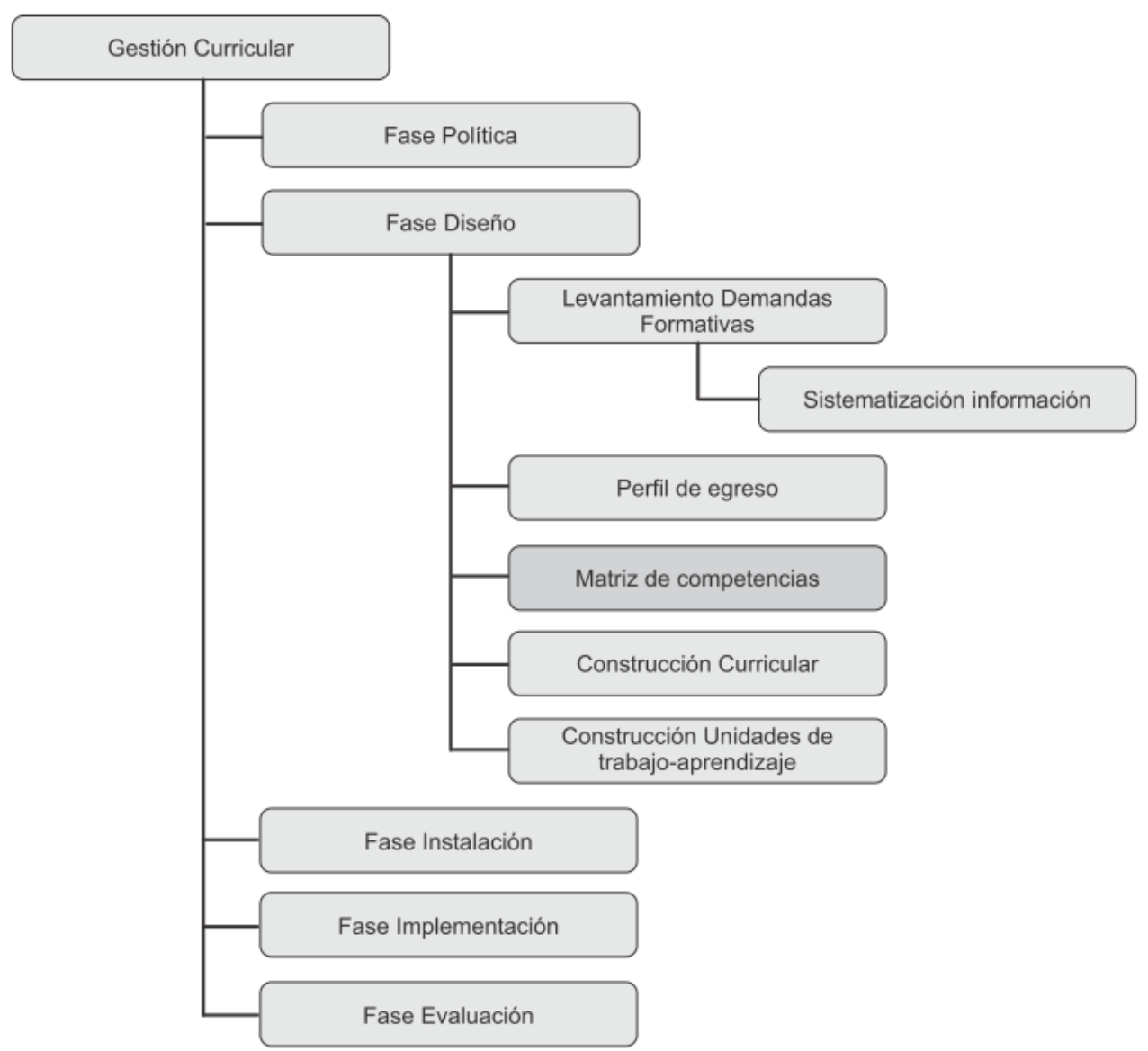

Fuente. (Tobón, 2008) citado en (Benavidez \& Vásquez, 2019)

Pero, como se mencionada, hay diversidad en esta noción relativamente reciente como lo es la gestión curricular. Otro modelo es el indicado por (V. Sánchez et al., 2016):

\section{- Etapa 1. Diagnóstico}

El objetivo en esta etapa es determinar las necesidades formativas de los profesionales a través de la evaluación de desempeño para la proyección de los planes y las políticas de desarrollo institucional, a fin de que se adquiera relevancia en el curso que le está asignado por su función transformadora. 


\section{- Etapa 2. Proyección}

Se busca el desarrollo de acciones que propicien la transformación de los modos de actuación de los actores involucrados, su crecimiento profesional y humano y el incremento de las capacidades que requieren las metas fijadas. La configuración y organización de vínculos de participación curricular comprende organizar y proyectar las acciones de capacitación e integración, de forma tal que se geste la coparticipación.

\section{- Etapa 3. Metodología}

Intervención curricular: comprende la acción curricular efectuada de forma conjunta por los actores del proceso en todos los componentes curriculares que están conectados a través de relaciones sistémicas que explican la naturaleza de los vínculos internos y externos.

\section{- Etapa 4. Evaluación}

Los actores evalúan las decisiones adoptadas en cada etapa y momento de este, a través de las actividades metodológicas, lo que permite su perfeccionamiento.

Entre sus objetivos están:

- Evaluar la transformación del proceso de gestión curricular a través de los resultados obtenidos por los egresados en sus cursos de superación.

- Evaluar la estrategia de gestión curricular a través de una guía de evaluación de desempeño que propicie la valoración de su actuación profesional.

\section{Cambios en la currícula}

Es fundamental pensar en la necesidad del alumno de mejorar, durante la formación profesional, los conocimientos y habilidades capaces de dar seguridad al proceso de cuidado, así como el desarrollo de habilidades de gestión con el fin de estimular las habilidades de motivación y liderazgo. hacia el trabajo en equipo, que son elementos capaces de dar excelencia al ejercicio profesional (Da Silva et al., 2018), sobre todo en tiempos como los actuales en el que se requieren cambios fundamentales en los procesos de enseñanza-aprendizaje para hacer frente a la Covid-19 (J. Sánchez et al., 2019). 
Mg. Mistral Ena Carhuapoma Acosta, Dra. Angela Rocio Cornejo Valdivia

La educación, en particular la universitaria, hace pertinente las modificaciones curriculares que analicen el trasfondo de los escenarios actuales, los que tensionan y conflictúan los modos tradicionales necesarios para estructurar y configurar la arquitectura curricular (J. Sánchez et al., 2019).

Desde el concepto de currículo, es posible identificar tres perspectivas al momento de generar cambios en este: 1) Como Construcción social, es decir como puente entre la sociedad y la escuela, donde se ve el currículo por objetivos y competencias; 2) Como proyecto educativo, en el que se representan los contenidos y las secuencias; y, 3) Como una unidad en la que convergen diferentes prácticas que no son solo de carácter pedagógico, sino que se consideran las interacciones y relaciones con el contexto (Benavidez \& Vásquez, 2019).

Emergen temas como la innovación curricular, (Poveda \& Gil, 2017) la consideran el valor de mayor aporte, para estos, la gestión curricular es "comprendida como un conjunto de procesos, decisiones y prácticas para estimular, dinamizar, desarrollar y reflexionar en torno al currículo y su buena práctica. También la implementación de competencias y una buena docencia, estos cambios son difíciles y profundos, sobre todo actualmente donde los alumnos deben adaptarse rápidamente y en momentos en los que se valoran mucho más el acompañamiento y la experiencia clínica del docente (Troncoso et al., 2017).

Si bien a nivel de las instituciones, las urgencias y la rapidez de actuación que impuso la pandemia obligó a una capacitación exprés del personal de enfermería, ahora toca hacer una reestructuración, incorporando las lecciones aprendidas de cara próximos eventos. La pandemia de Covid-19, ha supuesto que el curso 2020-2021 del Grado en Enfermería empiece a plantearse tras muchos cambios organizativos y curriculares respecto a años anteriores, este es una necesidad y urgencia en la medida que supone una transformación en la formación del estudiantado (Belén et al., 2020). Algunos de esas modificaciones incorporando tecnología ya se ha venido dando y entre los ingresos más significativos puede mencionarse los siguientes:

\section{Modelos sincrónicos y asincrónicos}

El factor tiempo resulta crucial en la medida en que invita a repensar y, por ende, redefinir, el conjunto de competencias, contenidos y logros de aprendizaje de los programas formativos de los distintos grados (Tejedor et al., 2020) en el nuevo contexto. 
Marques, 2020(Marques, 2020) señala que deben combinarse, en la actualidad, los enfoques utilizando interacciones sincrónicas y asincrónicas, como lo recomendaron otros autores (Watts, 2016). Las clases en línea, por ejemplo, menciona que se pueden llevar a cabo en bloques de dos o tres horas en una web con el video en vivo sincrónico a través de un servicio de videoconferencia basado en la nube. Este método permite la interacción entre estudiantes y profesores para mejorar el compromiso.

Por otro lado, algunas clases se pueden impartir con la utilización del modo asíncrono (presentaciones de PowerPoint de grabación de voz) para permitir la autonomía y la flexibilidad, se evitó un posible conflicto de programación con compromisos familiares/profesionales debido al bloqueo o en el caso de dificultades técnicas. Además, permitió a los estudiantes revisar el contenido y reforzar sus estudios.

Esto se relaciona con lo indicado por Tejedor et al., 2020 quien acotó que en el escenario de la 'nueva normalidad' se ha incrementado la importancia de la gestión del tiempo, la autonomía y la flexibilidad en la formación de los futuros profesionales.

Cabe mencionar, no obstante, lo dicho por (Feyen, 2020), quien enfatiza que la implementación exitosa de clases virtuales no solo requiere de la disponibilidad de la tecnología, sino también que el personal académico reciba capacitación en enseñanza en línea, sobre el desarrollo de material digital del curso y la capacitación de los estudiantes durante las sesiones en línea y de trabajo.

\section{TICs en la educación}

Arandojo, 2016 comenta que las denominadas Tecnologías de la Información y Comunicación (TICs) se han revelado como instrumentos que refuerzan, ayudan y mejoran el actual modelo sanitario y son los enfermeros los que ha comenzado a hacer uso de ellas a fin de garantizar la atención, cobertura y continuidad de los cuidados, mejorar los procesos de comunicación y lograr adecuar los recursos sanitarios disponibles a las demandas existentes

Zarate et al, 2020, también indica que elementos como las TICs permitieron optimizar la didáctica en el proceso enseñanza aprendizaje de los alumnos de diversas instituciones en tiempos de Covid-19.

La educación virtual y en línea es una realidad a medida que el ser humano ingresa diariamente (y cada vez con más profundidad) a la llamada sociedad de red del siglo XXI. 
En la actualidad es más accesible y considerada por muchos como la mejor opción frente al sistema educativo tradicional de universidades y colegios presenciales. En general, se acepta que la educación digital crea nuevas oportunidades de aprendizaje a medida que los estudiantes participan en entornos digitales en línea y los maestros cambian las prácticas educativas mediante el uso de cursos híbridos, instrucción personalizada, nuevos modelos de colaboración y una amplia gama de estrategias de aprendizaje innovadoras y atractivas (Feyen, 2020).

No obstante, autores como (Zárate et al., 2020) señalan que se requieren estrategias y cambios en el método de enseñanza, la adaptación de nuevos modelos para dar cumplimiento al currículum o plan de estudios, y al ser una disciplina y profesión donde la mayor parte de los conocimientos se basan en el desarrollo de competencias y habilidades prácticas, es necesario repensar, modificar el modelo tradicionalista por uno ecléctico para cumplir con los objetivos de aprendizaje.

\section{Revaloración de la Simulación clínica}

La simulación clínica se ha consolidado como un componente importante del currículo universitario en las ciencias de la salud, y ha demostrado ser útil para la evaluación objetiva de las competencias que deben tener los profesionales de esta área. La simulación se refiere a la aplicación de procesos simulados en objetos para la educación y formación de estudiantes.

Parte necesaria para el aprendizaje por este medio es contar con un escenario cuyo propósito sea proporcionar el contexto en el que se llevarán a cabo las actividades. Estatécnica, empleada de manera formal en la educación de enfermería desde hace más de 20 años, ha permitido un mejor adiestramiento de estudiantes de enfermería y de especialistas en diferentes áreas clínicas y perfeccionamiento de técnicas invasivas y quirúrgicas (Zárate et al., 2020).

Dentro de esta nueva realidad causada por la pandemia de la Covid-19 en la cual el estudiante de enfermería no puede realizar prácticas clínicas, se podría considerar como un elemento central a la simulación clínica para que los estudiantes puedan ejercitar y recapacitar sobre su rendimiento, con la finalidad de estar acordes a las nuevas exigencias, con las destrezas y habilidades clínicas para el trabajo en equipo. La simulación y el debriefing ofrecen un ambiente seguro para reflexionar sobre nuevos conceptos, realizar 
nuevas prácticas y desarrollar nuevos mapas mentales, mejora así la toma de decisiones y el trabajo colaborativo (Ayala \& Espinoza, 2020).

Por todo ello, la simulación clínica que es considerada como una revolución en la formación médica, lleva la práctica a un escenario lo más parecido a la realidad, pero sin riesgo para el paciente y con menores implicaciones éticas (Daniel et al., 2019).

\section{Conclusiones}

En la medida que la presente se propuso como objetivo reflexionar sobre la situación panorámica de la gestión curricular en programas de enfermería en el contexto de la pandemia por Covid-19, ello a través de la revisión de la literatura de los últimos años y el respectivo análisis de los artículos identificados, se puede indicar el cumplimiento de este fin.

A lo largo de la literatura se ha visto un cambio en la educación sobre todo la de instituciones superiores en todo el mundo, asociados al aislamiento social obligatorio y el resguardo de la vida a través de diversas medidas de restricción en las interacciones personales. Ello afectó directamente a la educación, sobre todo debido a la falta de recursos y metodologías innovadoras con las que muchas casas de estudios no contaban.

A su vez, la pandemia hizo relevante las diferencias y contrastes en la brecha digital y de recursos de la mayoría de los alumnos en países en vías de desarrollo como es el caso de Perú y más aún en universidades estatales.

Ello no es ajeno a la disciplina de la enfermería, la cual se vio envuelta en un contexto incierto y de muchas dudas respecto a cómo actuar con éxito para que los alumnos tengan programas de calidad sin ponerlos en riesgo.

Se necesita, por ello, el trabajo colaborativo con otras Escuelas de Enfermería, abrir las puertas de la creatividad que caracteriza la respuesta del ser humano en tiempos complejos, así también solidaridad interdisciplinar y, por supuesto, apoyo de políticas públicas que respondan, a corto plazo, a las brechas señaladas y permitan entregar formación de calidad, humana y equitativa.

Si bien la tecnología e innovación son elementos clave en tiempos de pandemia, lo es también el repensar y reorientar los contenidos de los programas de enfermería para que tanto las casas de estudios como los estudiantes cuenten con los cimientos necesarios 
ya sean saberes y recursos materiales para hacer frente a otras situaciones semejantes que se presentarán, sin duda, en el futuro.

El escenario actual puede tornarse especialmente fértil para que los países inviertan en mejorar las condiciones de trabajo y educación en enfermería, lo cual redundará en importantes logros para la cobertura universal de salud y el acceso a la salud de las poblaciones de la Región.

\section{Referencias}

Álvarez, M., \& Díaz, H. (2019). Gestión curricular de la educación superior en ciencias de la salud. Pontificia Universidad Javeriana. https://repository.javeriana.edu.co/bitstream/handle/10554/155/edu23.pdf?seque nce $=4 \&$ isAllowed $=y$

Ángel, G., \& Lopera, C. (2013). Relevance y level of application of management competencies in nursing. Investigación y Educación En Enfermería, 31(1), 0919.

Ayala, D., \& Espinoza, T. (2020). Utilidad de la simulación clínica para lograr competencias en estudiantes de enfermería en tiempos de COVID-19. Revista Cubana de Enfermería, 36, 1-16.

Belén, A., Valera, S., Carmen, A., López, L., Solanas, I. A., Urcola, F., José, P., Dorda, S., \& Gracia, A. Á. (2020). Estudio mixto del distrés psicológico en los alumnos del Grado en Enfermería ante las prácticas curriculares externas durante la pandemia de Covid-19. Paraninfo Digital Proyecto, XIV(32), 1-4.

Benavidez, M., \& Vásquez, L. (2019). La importancia de la gestión curricular universitaria en programas a distancia, estudio Institución de Educación Superior Suramericana. Revista In Crescendo, 10(1), 13-34.

Cassiani, S., Munar, E., Umpiérrez, A., Peduzzi, M., \& Leija, C. (2020). La situación de la enfermería en el mundo y la Región de las Américas en tiempos de la pandemia de COVID-19. Revista Panamericana de Salud Publica/Pan American Journal of Public Health, 44, 1-2. https://doi.org/10.26633/RPSP.2020.64

Costa, R., Motta, M., Jatobá, A., Lorenzini, E., Manfrini, G., Farias, L., Oliveira, M., Honório, M., \& Gonçalves, N. (2020). Enseñanza de enfermería en tiempos de 
covid-19: ¿cómo reinventarla en este contexto? Texto \& Contexto - Enfermagem, 29, 1-3. https://www.scielo.br/pdf/tce/v29/es_1980-265X-tce-29-e20200202.pdf

Da Silva, A. M., Celich, K. L. S., Da Silva, T. G., De Souza, S. S., Bittencourt, J. V. V., \& Godinho Bertoncello, K. C. (2018). Nurses' perception towards the teachinglearning process of Nursing management / Percepção de enfermeiras quanto ao ensino e aprendizagem da gestão em Enfermagem. Revista de Pesquisa: Cuidado é Fundamental Online, 10(4), 1098. https://doi.org/10.9789/21755361.2018.v10i4.1098-1102

Daniel, A., Domínguez, G., Andrade, A., \& Morales, S. (2019). High fidelity simulation and pause reflection method in medical students of the UNAM. Educacion Medica, xx. https://doi.org/10.1016/j.edumed.2019.02.011

Elseiver, \& R\&A Smarth Health. (2020). Enfermería durante la pandemia: retos, oportunidades y lecciones aprendidas.

Feyen, J. (2020). Shall COVID-19 accelerate the transfer of passive learning to active education? Maskana, 11(1), 1-4. https://doi.org/10.18537/mskn.11.01.00

González, G., Bores, D., Barba, R. A., \& Gallego, V. (2020). Learning to be a teacher without being in the classroom: COVID-19 as a threat to the professional development of future teachers. International and Multidisciplinary Journal of Social Sciences, 9(2), 152-177. https://doi.org/10.17583/rimcis.2020.5783

Guzman, A. (2013). Gestión curricular: la experiencia de la Licenciatura en Enfermería de la Universidad de Costa Rica. Revista Electrónica Enfermeía Actual En Costa Rica, 24, 1-14. http://www.revenf.ucr.ac.cr/gestion.pdf

Huanca, J., Supo, F., Sucari, R., \& Supo, L. (2020). El problema social de la educación virtual universitaria en tiempos de pandemia, Perú. Innovaciones Educativas, 22(Especial), 115-128. https://doi.org/10.22458/ie.v22iespecial.3218

Jaque, R. (2020). Nursing education in pandemic times: Present and future challenges. Ciencia y Enfermeria, 26, 1-3. https://doi.org/10.29393/CE26-14EERL10014

Marques, P. (2020). Uso de lecciones sincrónicas y asincrónicas para la educación de enfermería durante la pandemia de COVID-19. 1-3. 
Mg. Mistral Ena Carhuapoma Acosta, Dra. Angela Rocio Cornejo Valdivia

Poveda, A., \& Gil, H. (2017). La ruta hacia la innovación social. AXIOMA - Revista Científica de Investigación, Docencia y Proyección Social, 16, 49-59.

Ríos, C. (2020). COVID-19 y Educación Superior Universitaria Pública del Perú. Revista Clake Education, $1(2)$, $1-1$. http://revistaclakeeducation.com/ojs/index.php/Multidisciplinaria/article/view/16 15

Sánchez, J., Morales, C., \& Burgos, M. (2019). Processes and strategies of curricular innovation in the nursing major, Arturo Prat University, Chile. Revista Cubana de Educacion Medica Superior, 33(2), 1-20. http://www.revenf.ucr.ac.cr/gestion.pdf

Sánchez, V., Izquierdo, J., Burgal, C., \& Charón, K. (2016). Estrategia de gestión curricular para tecnólogos de la salud del perfil en Administración y Economía de la Salud. Medisan, 20(5), 708-717.

Speranza, S., Recalde, N., \& Paciello, M. A. (2020). Valores en época de coronovirus (Covid-19) en Paraguay. Academic Disclosure Una Fenob, 252-261.

Tejedor, S., Cervi, L., Tusa, F., \& Parola, A. (2020). Educación en tiempos de pandemia: reflexiones de alumnos y profesores sobre la enseñanza virtual universitaria en España, Italia y Ecuador. Revista Latina, 78, 1-21. https://doi.org/10.4185/rlcs2020-1466

Tobón, S. (2008). La formación basada en competencias en la educación superior: el enfoque complejo. México: Universidad Autónoma de Guadalajara, 20(December), $1-30$. http://biblioteca.udgvirtual.udg.mx/jspui/handle/123456789/3491

Troncoso, M., Gonzalez, C., Guerrero, V., Gonzalez, C., Vasquez, P., \& Rojas, A. (2017). Nursing students' experiences of change: from a traditional curriculum to a competency-based curriculum. Revista de Enfermagem Referência, IV Série(No14), 49-56. https://doi.org/10.12707/riv17036

Velazque, L., Valenzuela, C., \& Murillo, F. (2020). Pandemia COVID-19: repercusiones en la educación universitaria. Odontología Sanmarquina, 23(2), 203-205. https://doi.org/10.15381/os.v23i2.17766 
Watts, L. (2016). Synchronous and Asynchronous Communication in Distance Learning: A Review of the Literature, Quarterly Review of Distance Education, 2016. ERIC, 17(1), 23-32. https://eric.ed.gov/?id=EJ1142962

Zárate, R., Ostiguín, R., Castro, A. R., \& Valencia, F. (2020). ENFERMERÍA Y COVID19: la voz de sus protagonistas. Universidad Nacional Autónoma de México. https://www.aladefe.org/noticias/Enfermeria_y_Covid.pdf 